\title{
The Pandemic's Impact on Digital Platform Businesses
}

\author{
Oksana Gerwe (Brunel Business School, Brunel University London) \\ Rosario Silva Froján (IE Business School)
}

KEYWORDS: Information Technology, Sharing

Economy.

The $21^{\text {st }}$ century brought about an explosion of businesses built around digital platforms, which transformed markets and economies worldwide. The socalled platform revolution upended entire industries from retail (Alibaba, eBay), deliveries (Amazon), online payments (PayPal), food delivery (Just-Eat, FoodPanda, Deliveroo), freelance jobs (Freelancer, Upwork) to transportation (Uber, Blablacar) and accommodations (Airbnb). Until recently, the success of digital platforms seemed almost unstoppable, as continuously manifested by the number of suppliers, consumers, and transactions on such platforms. However, the Covid-19 pandemic made it painfully obvious that not all platform businesses are equally resilient when a global crisis hits. As Warren Buffett famously noted, "only when the tide goes out do you discover who's been swimming naked."

The success of digital platforms has been predicated on the use of information technologies to efficiently connect and match suppliers with consumers. For example, an Airbnb property owner in Sydney can now be easily matched with a German tourist looking for accommodations in Australia, which would have been very difficult to arrange in the past. In this case, the platforms serve as facilitators or intermediaries of transactions between the two sides: setting the rules, providing the IT backbone, safe payment, and generating mechanisms of digital trust in the transaction. For example, Uber drivers and passengers mutually rate each other, which creates greater transparency and ensures quality in a peer-to-peer transaction.

However, the Covid-19 pandemic brought about abrupt changes in the market environment and the economy at large. Shifts in demand and the radical measures taken by governments to combat the spread of the novel coronavirus created massive disruption in the business landscape with particular relevance for digital platforms.

\section{A Disruptive Force}

On the one hand, digital platforms have helped alleviate the negative situation, enabling individuals and small businesses to maintain their activity in the digital landscape and allow consumers to access goods and services even in a situation with strong movement restrictions. For example, deliveries of goods, meals or groceries by Amazon, Deliveroo or Ocado, respectively, skyrocketed during the lockdown months. Also, compared to traditional businesses, digital platforms found themselves better positioned to come up with more up-to-date and well-informed strategies to combat the crisis. Indeed, platforms by nature accumulate large amounts of data from their suppliers, customers and transactions that can help the company reorient its strategies. Hence, their decision-making can be superior to some of the traditional businesses, which lack access to vast amounts of relevant data on their business operations, or lack the ability to make sense of it. Finally, because these platforms have few physical assets, they have unparalleled flexibility to scale quickly to take advantage of opportunities or reduce their operations without collapsing.

\section{Winners and Losers}

On the other hand, not all digital platforms performed equally well during the current crisis. Several factors separated platform businesses that benefited from the Covid-19 pandemic from those that suffered from it. First, the introduction of lockdowns and shelter-in-place orders made transactions impossible for all those platforms that relied on freedom of movement and close face-to-face interactions between suppliers and consumers. For example, taking a ride through ridehailing apps of Uber, Lyft or BlaBlaCar became impossible because people could not leave their homes. Besides, the need to share a ride with another person (a driver) created very real risks to the health and safety of both suppliers and consumers of services offered by a range of digital platforms.

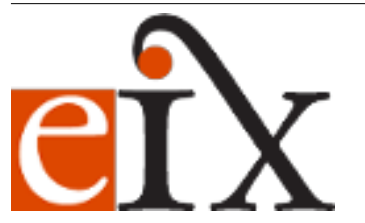

Copyright ( ) 2021 The Authors. Entrepreneur \& Innovation Exchange is published at EIX.org. This is an open access article under the terms of the Creative Commons Attribution-NoDerivs License, which permits use and distribution in any medium, provided the original work is properly cited and no modifications or adaptations are made. View EIX.org Authorship Terms at https://eix.org/terms 
In contrast, platforms that did not need close contact between consumers and suppliers, such as food deliveries, online retail, etc., saw a huge boost from the lockdowns and new standards for health and safety. That is why platforms such as Amazon, Deliveroo and eBay saw a sharp rise in transactions from March 2020 through the end of the year. Second, some platforms came into the pandemic overloaded with inflated losses. Thus, at the first sign of the drop in demand, we saw a major shift towards aggressive cost-cutting. For example, Uber fired a substantial part of their workforce and undertook other cost-saving strategies in order to salvage their balance sheets. Over the last decade, many platforms pursued the strategy of growth over profitability. The pandemic served a dose of bitter medicine to bring their focus back on business fundamentals, which is likely to have a positive effect on the operations of these companies in the long run. The WeWork platform, for example, even prior to the pandemic suffered major losses due to an overaggressive growth strategy and as a result had to cancel IPO plans in 2019. Now, in the midst of Covid-19 crisis, the company is fighting for its survival but its business prospects are unclear. Finally, some platforms suffered less during the crisis because they diversified their sources of revenue. For instance, Uber's presence in food delivery has mitigated the decline in revenues of the ride-hailing business.

\section{A Chance to Re-focus}

With a number of vaccines awaiting regulatory approval, the end of the pandemic is hopefully in sight. We can't predict with certainty which effects of the coronavirus crisis will be temporary and which will persist. But it is fair to say that, just like any other crisis, the Covid-19 pandemic has created both profound challenges and extraordinary opportunities. For example, the promise of the economic recovery has already pushed up the valuation for Airbnb. In the biggest IPO of 2020 in the USA, the company's value almost doubled, reaching about $\$ 100$ billion in December 2020. Similarly, in the UK, Deliveroo is considering an IPO in early 2021.

Digital platform businesses with their goods and services have undoubtedly helped many of us get through the disconnect, isolation and shortages of the pandemic months. Despite their differences, digital platforms have proven to be more resilient than many traditional businesses in facing the challenges of an extreme crisis. But, while some platforms have reinforced their central position in the economy, others have struggled to maintain their business. Let's hope that the bitter pill of Covid-19 helps the negatively affected platforms regain their strategic focus, prioritize business fundamentals over uncontrolled growth, and maintain a lower cost structure not only during the current crisis but also in the post-pandemic recovery period, leading to higher margins and greater profitability. 